\title{
Análisis del cumplimiento de la atención fonoaudiológica de la guía clínica GES, en pacientes con enfermedad de Parkinson en centros de atención primaria de salud de Talca Analysis of compliance phonoaudiologic care of clinical guide GES in patients with Parkinson's disease in primary care centers health Talca
}

Nancy Ferrada R. ${ }^{1}$, Natalia Catalán C. ${ }^{2}$, Gonzalo Dachelet G., Gemima Muñoz Ll. ${ }^{2}$ y Alex Soto P. ${ }^{3}$

Since 2010 the Chile's Ministry of Health includes Parkinson's disease (PD) pathology in AUGE. Accordingly, this unit produced a clinical guideline wherein stipulates a specific procedure for assessment and intervention in Primary Care Health Centers. In that guideline, the speech therapy intervention is indicated as fundamental, applicable in a given amount of annual sessions depending on the phase of the disease for each person. Currently there are 518 cases in control in the province of Talca. Therefore, the present investigation aims to establish the level of accomplishment of speech therapy services in Parkinson's disease as required by GES law in Primary Care Health centers of the city of Talca.

Key words: Parkinson disease, speech therapy, primary health services. Rev Chil Neuro-Psiquiat 2013; 51 (2): 110-114

\section{Introducción}

$\mathrm{L}$ a (EP) corresponde a una patología neurológica progresiva y crónica que no se cura y empeora gradualmente (Weiner, Shulman y Lang, 2001). La evolución de la Enfermedad de Parkinson se desarrolla lentamente, en donde ha transcurrido varios años de muerte neuronal (Juri et al, 2004). Las enfermedades neurodegenerativas afectan no sólo al individuo que la padece, sino también a aquellos que lo rodean. Implica una serie de trastornos psico-emocionales, económicos y sociales que de- ben ser conocidos y asumidos por el paciente y la familia. En este contexto, una de las enfermedades neurodegenerativas frecuentes en Chile es la Enfermedad de Parkinson, aspecto que se relaciona con el aumento en la tasa de envejecimiento de nuestro país (MINSAL, 2010). Tal antecedente generó que en el año 2010 se incorpore al Regimen de Garantías Explicitas en Salud (GES) dicha enfermedad (MINSAL, 2010a). La ley GES No 19966 es parte importante de la reforma de salud de Chile puesta en marcha el año 2005 (MINSAL, 2004). Entre otras cosas esta reforma pretende estandarizar

Recibido: 27/02/2013

Aceptado: 27/05/2013

Enfermera, Magíster en Gestión de Instituciones de Salud. Escuela de Fonoaudiología Universidad de Talca.

Estudiante, Escuela de Fonoaudiología Universidad de Talca.

Estadístico, Departamento de Estadística y Matemáticas Universidad de Talca. 
la atención de las patologías contenidas en ella y asegurar acceso, calidad, oportunidad y apoyo financiero a los pacientes que la padecen.

Con el apoyo de expertos se desarrollan Guías Clínicas para estas patologías, destinadas a dar una orientación a los profesionales de la red pública y privada de salud, para tratar a los pacientes según el nivel de su patología. En esta guía se encuentran los procedimientos a realizar en el diagnóstico, tratamiento y monitoreo de la enfermedad, como también el equipo interdisciplinario de profesionales que participan en el manejo del paciente. Según Soto (2009), la atención primaria incorpora a profesionales, entre ellos el Fonoaudiólogo, cómo parte de este grupo interdisciplinario, entregando una atención integral, consecuente con el nuevo modelo de reforma de la salud.

La EP se incorpora como objeto de estudio en este proyecto de investigación. Dicha enfermedad, es un proceso neurodegenerativo progresivo. Según la guía clínica GES de EP (2010b), la lesión fundamental de la EP recae en la parte compacta de la sustancia negra, que forma parte de los ganglios basales, componentes del sistema nervioso. Al describirse como una afección neurodegenerativa, se afectan progresivamente, diferentes procesos entre los que se encuentra el habla y la voz (Estevos et al 2003). La deglución es otra de las funciones afectadas (Wallace, Schwartz, DeCaele, Zagami y Cook, 1996; Bleeckx, 2004). Sobre las alteraciones que se presentan en estas áreas, es la Fonoaudiología la disciplina científica a cargo de su evaluación, diagnóstico y tratamiento.

En la Guía Clínica GES de Enfermedad de Parkinson (MINSAL, 2010b), se dicta el diagnóstico oportuno y posterior inicio de tratamiento en un transcurso de no más de 20 días. Así también, se especifica la cantidad de sesiones de evaluación, intervención y reevaluación que deben ser realizadas por equipo multidisciplinario de salud entre los que se encuentran Médicos, Kinesiólogos, Psicólogos, Terapeutas Ocupacional y Fonoaudiólogo en los centros de atención primaria de salud.

Resulta importante determinar la consecuencia de lo establecido por el MINSAL con los hechos reales acontecidos y así poder establecer una conclusión que esté disponible para el trabajo, discusión y elaboración de proyectos en pro de la comunidad y sociedad.

De lo anterior se desprende el propósito de este proyecto de investigación, cuyo fin es establecer el nivel de cumplimiento de la atención fonoaudiológica en la Enfermedad de Parkinson según lo establecido por la ley GES en Centros de Atención Primaria de Salud de la ciudad de Talca.

\section{Método}

Esta investigación se realizó en cuatro de los siete centros de Atención Primaria en Salud (APS) de la ciudad de Talca, perteneciente a la región del Maule, Chile. Sólo se consideraron los centros que cuentan con recurso de Fonoaudiología, ya sea profesionales o internos de la especialidad.

El estudio es descriptivo no experimental transeccional. La muestra es no probabilística y correspondió a 35 sujetos con EP, 13 mujeres y 22 hombres, entre edades de 52 y 85 años.

La estimación poblacional del tamaño muestral fue establecida a través del uso del programa informático de cálculo GRANMO versión 7 (2012), donde se establecen los datos ingresados, obteniendo lo siguiente:

Aceptando un riesgo alfa de 0,95 para una precisión de \pm 20 unidades en un contraste bilateral para una desviación estándar estimada de 80 , se precisa una muestra de 34 sujetos, asumiendo que la población es de 58 sujetos. Para la inclusión de los sujetos al estudio, se considerará a aquellos que estén registrados durante el período 2010-2012 en los centros de atención primaria de salud y que firmen el consentimiento informado brindado por el equipo investigador.

Se excluyeron aquellos pacientes que no se encontraron registrados en los centros de atención primaria o que su registro sea anterior al año 2010. Los sujetos que participaron en este estudio, corresponden a ocho del centro de salud las Américas, quince del centro Julio Contardo, ocho del centro Carlos Trupp y cuatro del centro Faustino González.

El protocolo de estudio fue aprobado por el 
comité de Bioética de la Universidad de Talca y autorizado por la Dirección Comunal de Salud de Talca. Luego se contactaron a los pacientes, se solicitó su participación y se incorporaron sólo los que firmaron el consentimiento informado.

Los datos fueron obtenidos mediante la revisión de cada ficha clínica y formulario GES de los pacientes con diagnóstico de EP. Luego de registrar los datos, se codificaron y se procedió a su análisis mediante el programa SPSS en su versión 19.

\section{Resultados}

Según los resultados obtenidos, el mayor número de sujetos con EP pertenecen al Centro de Salud Familiar (CESFAM) Julio Contardo con un porcentaje del $43 \%$ equivalente a 15 sujetos, seguido por los CESFAM Las Américas y Carlos Trupp, ambos con 8 sujetos, equivalente a un 23\% para cada centro. Finalmente, el Centro Comunitario de Salud Familiar (CECOF) Faustino González con un porcentaje de un $11 \%$ correspondiente a 4 individuos de la muestra.

El mayor número de pacientes con EP pertenece al rango de edad entre los 70-79 años, que correspondiente al 37\%, seguido del rango 60-69 años con un 32\% y el rango 50-59 años que presenta un $17 \%$. El rango de menor porcentaje, es el de 80-89 años, con un $14 \%$. Existe una concordancia entre los resultados obtenidos y lo que destaca la Guía Clínica, que menciona el inicio de la EP fluctuante entre los 50 y 60 años (MINSAL, 2010b).

Finalmente, es importante considerar que al aumentar la edad, crece también la posibilidad de padecer EP (Chaná, 2010).

Se establece que del total de la muestra, el 63\% pertenece al género masculino y el $37 \%$ a femenino. Los datos corroboran lo que explica Chaná (2010), quien menciona que la incidencia de la enfermedad parece ser mayor en varones, entre 1,5 y 2 veces más que en mujeres.

$\mathrm{La}$ atención interdisciplinaria que reciben los pacientes con EP según centro de atención de salud corresponde a:

CESFAM Julio Contardo. De los 15 pacientes correspondientes a la muestra, la totalidad de ellos re- ciben atención médica y tratamiento farmacológico. El 53\% de los pacientes reciben además intervención kinésica, mientras que el 27\% además de la atención médica y kinésica reciben intervención psicológica. Tan sólo el 7\% del total, junto a los tratamientos anteriores, son beneficiarios también de la intervención de un terapeuta ocupacional. Por otro lado, existe un 13\% que sólo recibe intervención médica, sin la participación de algún otro profesional de la salud, indicado por la guía clínica de EP para el manejo de los sujetos con dicha enfermedad.

En el centro CESFAM Carlos Trupp, grupo compuesto por 8 sujetos, el 13\% (1 sujeto) recibe sólo tratamiento médico y farmacológico. El 87\% restante (7 sujetos) reciben tratamiento médico, kinesiológico y de terapia ocupacional. Se destaca que no existen sujetos que posean registros de asistencia psicológica durante el tratamiento de la EP.

En el CESFAM Faustino González se desprende que el 25\% de los sujetos recibe atención médica y kinesiológica, el 50\% atención médica, kinesiológica e intervención psicológica y el 25\% restante además recibe intervención de terapia ocupacional.

En cuanto a la intervención fonoaudiológica que reciben los pacientes con EP, considerándose proceso de evaluación, tratamiento y reevaluación, de manera independiente o conjugada, se observa un porcentaje mayoritario de un $91 \%$, equivalente a 32 sujetos que no reciben intervención fonoaudiológica. Un 3\%, correspondiente a un sujeto, sólo recibe evaluación fonoaudiológica. Asimismo es 1 sujeto $(3 \%)$ que recibe sólo tratamiento fonoaudiológico. Por último, el 3\% de la muestra, también equivalente a 1 sujeto, es beneficiario tanto de evaluación como de tratamiento.

En el CESFAM Las Américas se observa que el $87 \%$, equivalente a 7 sujetos, no recibe algún tipo de intervención fonoaudiológica. Mientras que existe 1 sujeto de que no se registran datos, correspondiente al 13\% del total.

Por otra parte, en el CESFAM Julio Contardo de un total de 15 sujetos con EP un 93\%, no recibe ningún tipo de intervención fonoaudiológica y tan sólo 1 sujeto equivalente al $7 \%$ es derivado a evaluación fonoaudiológica.

Continuando con el análisis por centro, en el 
CESFAM Carlos Trupp, un $87 \%$ de la muestra, equivalente a 7 sujetos no recibe ningún tipo de intervención fonoaudiológica. Por su parte, es sólo 1 sujeto, conformante del 13\% que recibe intervención fonoaudiológica tanto de evaluación como de tratamiento, siendo ambas de manera conjunta. Por consecuencia, se aprecia un porcentaje significativo que no recibe intervención fonoaudiológica, de ningún tipo anteriormente indicado.

Por último en el CECOF Faustino González, la distribución de relación fonoaudiológica que allí se presenta. Se aprecia, que del total de sujetos, equivalente a cuatro, el $100 \%$ no recibe ningún tipo de intervención fonoaudiológica.

Como resultado el 91\% de la muestra, equivalente a 32 sujetos, no reciben atención de ninguna de las áreas de intervención fonoaudiológicas ya especificadas. Por otra parte, se observa que un $6 \%$ del total correspondiente a dos sujetos, no registran datos en la ficha clínica sobre esta información. Mientras que un 3\% de la muestra, correspondiente a 1 sujeto, recibe atención de las áreas de audiología, habla y deglución, de modo simultáneo de acuerdo a lo registrado en su ficha clínica.

\section{Conclusiones}

Se puede observar en este estudio que la proporción de pacientes con EP que recibe atención fonoaudiológica es mucho menor a la que debiera ser atendida por esta especialidad, estando en discordancia con las recomendaciones contenidas en la Guía Clínica GES de esta patología. Esta diferencia puede deberse al desconocimiento de la misma por parte del personal médico a cargo del tratamiento de los pacientes o diferencias de criterio en la derivación. También puede deberse al desconocimiento de los pacientes de la patología y su tratamiento. Además existe desinformación respecto al quehacer profesional fonoaudiológico en las áreas de habla, voz y deglución, como también de sus derechos y beneficios contenidos en esta guía, razón por la cual no exigen ni solicitan la atención de éste y otros profesionales recomendados. Sin embargo, se requiere otro estudio más detallado que pueda dar cuenta de cuáles son los factores que influyen en la falta de atención de estos pacientes.

Se sospecha que dicho desconocimiento se debe principalmente a que la mayoría de los sujetos de la muestra, no recibe orientación adecuada a su patología y del tratamiento propuesto por la GES.

Otro de los factores que puede explicar el fenómeno es la falta de profesional Fonoaudiólogo en los centros de atención, pues sólo uno de ellos, el CESFAM Carlos Trupp cuenta con $44 \mathrm{~h}$ de profesional. Este punto es discutible, pues en los otros tres centros si bien no existe profesional contratado, cuentan con internos de la especialidad y tampoco son derivados.

Se establece sin embargo, la presencia de tratamientos kinésicos, psicológicos y en menor grado de terapia ocupacional aunque no generalizados a toda la muestra y tampoco en la frecuencia recomendada por la Guía Clínica GES de la patología.

Al parecer el sistema de control de cumplimiento de las guías clínicas GES parece ser insuficiente para identificar las prestaciones entregadas a los beneficiarios.

De la información recopilada emana que no hay un cumplimiento de las recomendaciones de la Guía Clínica GES, por lo que es necesario evaluar las razones que expliquen esta situación.

Por último, es importante destacar que el Fonoaudiólogo es el especialista idóneo para realizar un proceso de rehabilitación en los pacientes con EP en cuanto a alteraciones en el habla, voz y deglución, puesto que dispone de los conocimientos y competencias al respecto. El objetivo fundamental de la intervención fonoaudiológica en pacientes con EP, es frenar el impacto de dicha patología, logrando en primera instancia mantener un proceso de alimentación con la menor cantidad de complicaciones posibles, para luego mejorar los parámetros vocales, junto con favorecer un habla inteligible, que permita una comunicación eficiente en diferentes contextos sociales. Debido a lo señalado, cobra real importancia para los centros de atención primaria de salud cuenten con profesionales fonoaudiólogos que les permita cumplir a cabalidad los procedimientos estipulados en la Guía Clínica GES para los sujetos con EP.

Por este motivo es necesario seguir estudiando 
para encontrar los factores y proponer las soluciones que sean permanentes en el tiempo, para que los pacientes con esta patología reciban todas las prestaciones a las cuales tienen derecho y se traduzcan en mejor calidad de vida para ellos y sus familias.

\begin{abstract}
Resumen
Desde el año 2010 el Ministerio de Salud incorpora la Enfermedad de Parkinson (EP) en las patologías AUGE. Para ello elaboró una Guía Clínica en donde se estipula un determinado procedimiento de evaluación e intervención para Centros de Atención Primaria de Salud. En ella, se indica la intervención fonoaudiológica como fundamental, aplicable en una determinada cantidad de sesiones anuales según el estadio de la patología en que se encuentra el sujeto. Actualmente en la provincia de Talca se encuentran 518 casos en control. Por consiguiente, la presente investigación tiene como objetivo establecer el nivel de cumplimiento de la atención fonoaudiológica en la Enfermedad de Parkinson según lo establecido por la ley GES en Centros de Atención Primaria de Salud de la ciudad de Talca.
\end{abstract}

Palabras clave: Enfermedad de Parkinson, fonoaudiología, atención primaria de salud.

\section{Referencias bibliográficas}

1. Ali GN, Wallace KL, Schwartz R, De Carle DJ, Zagami AS, Cook IJ. Mechanisms of oral-pharyngeal dysphagia in patients with Parkinson's disease. Gastroenterology 1996; 110: 383-92.

2. Bleeckx D. Disfagia, Evaluación y reeducación de los trastornos de la deglución. España: McGrawHill. 2004.

3. Calculadora de tamaño muestral granmo versión 7 abril de 2012. Extraído de http://www.imim.cat/ ofertadeserveis/software-public/granmo/

4. Estevo A, Papaterra J. Tratamento dos distúrbios da voz na doença de Parkinson. Revista Arq Neuropsiquiatría 2003; 61 (1): 61-6.

5. Chaná P. Enfermedad de Parkinson. Chile. Universidad de Santiago de Chile. CETRAM. 2010.

6. Chile. Aprueba Garantías Explícitas en la Salud del Régimen General de Garantías Explícitas en Salud $\mathrm{N}^{\circ} 1.2010$, de 06 de Enero del Ministerio de Salud. Publicado en el Diario Oficial 07 de Febrero de 2010.

7. Chile. Establece un Régimen de Garantías en Salud Ley 19.966/ 2004, de 04 de Septiembre del Ministerio de Salud. Publicado en el Diario Oficial 25 de Agosto de 2004.
8. Juri C, Rodríguez-Oroz M, Obeso J. Tratamiento de la enfermedad de Parkinson en estado inicial. Escuela de Medicina Pontificia Universidad Católica de Chile. Departamento de Neurología. 2004.

9. Ministerio de Salud (2010). Guía Clínica enfermedad de Parkinson. Santiago, Chile. Extraído de http://www.MINSAL.gob.cl/portal/url/item/9555 78f79a0cef2ae04001011f01678a.pdf

10. Ministerio de Salud (2010) OE ciclo vital Extraído de http://www.minsal.gob.cl/portal/url/item/94b4 cb1b29bc26a2e04001011e013430.pdf

11. Soto H. (2009). La fonoaudiología entra fuerte en la atención primaria. Santiago, Chile. Extraído de http://www.med.uchile.cl/entrevistas/2878-la-fonoaudiologia-entra-fuerte-en-atencion-primaria-. html

12. Weiner W, Shulman L, Lang A. (2001). "La enfermedad de Parkinson”. Una guía completa para pacientes y familia. Barcelona: Paidos, Página 19.

\section{Correspondencia:}

Mg. Nancy Ferrada Rojas

Clínica de Fonoaudiología, Universidad de Talca.

2 Norte 685, Talca Chile. Fono: 071- 2201600

E-mail: nferrada @utalca.cl 